\title{
Sur des variétés toriques non projectives
}

Bulletin de la S. M. F., tome 128, no 3 (2000), p. 407-431

<http://www.numdam.org/item?id=BSMF_2000_128_3_407_0>

(C) Bulletin de la S. M. F., 2000, tous droits réservés.

L'accès aux archives de la revue «Bulletin de la S. M. F. » (http: //smf.emath.fr/Publications/Bulletin/Presentation.html) implique l'accord avec les conditions générales d'utilisation (http://www.numdam.org/ conditions). Toute utilisation commerciale ou impression systématique est constitutive d'une infraction pénale. Toute copie ou impression de ce fichier doit contenir la présente mention de copyright.

\section{Numdam}




\title{
SUR DES VARIÉTÉS TORIQUES NON PROJECTIVES
}

\author{
PAR LAURENT BONAVERO $(*)$
}

\begin{abstract}
RÉSUMÉ. - Utilisant la théorie de Mori des variétés toriques projectives due à M. Reid, nous étudions les variétés toriques non projectives qui le deviennent après éclatement le long d'une courbe invariante.

ABSTRACT. - ON NON PROJECTIVE TORIC MANIFOLDS. - With the help of Mori theory for projective toric manifolds due to M. Reid, we study non projective toric manifolds which become projective after a single blow up along an invariant curve.
\end{abstract}

\section{Introduction}

Dans tout ce travail, nous appelons variété torique de dimension $n$ une variété torique complète et non singulière; autrement dit, nous nous intéressons aux compactifications lisses équivariantes du tore complexe $\left(\mathbb{C}^{*}\right)^{n}$. Une telle variété n'est en général pas projective, mais les exemples connus de variétés toriques non projectives peuvent être considérés comme isolés au sens où la cause géométrique de non projectivité n'est pas toujours comprise. Nous nous proposons ici d'étudier géométriquement les variétés toriques non projectives les plus simples. Pour cela, rappelons qu'il est bien connu (voir par exemple [Mor96]) que toute variété torique devient projective après une suite finie d'éclatements le long de sous-variétés lisses toriques; autrement dit, la version torique du théorème de Moishezon [Moi67] est vérifiée. Le but de ce travail est de comprendre les variétés toriques non projectives les plus simples à la lumière du théorème de Moishezon : celles qui deviennent projectives après éclatement le long d'une courbe torique (connexe) ( $X$ étant lisse, toute courbe torique de $X$ est rationnelle non singulière). Rappelons aussi que si $X$ est une variété complexe compacte et si $x$ est un point de $X$, alors $X$ est projective si et seulement si $B_{x}(X)$ est projective.

(*) Texte reçu le 3 juin 1999, révisé le 2 septembre 1999, accepté le 7 octobre 1999.

L. Bonavero, Institut Fourier, UFR de Mathématiques, Université de Grenoble 1, UMR 5582, BP 74, 38402 Saint Martin d'Hères CEDEx (France). Email : bonavero@ujf-grenoble.fr et http ://www-fourier.ujf-grenoble.fr/ bonavero/

Mots clés : variété torique, variété non projective, théorie de Mori.

Classification mathématique par matières : 14M25, 14E05, $14 \mathrm{E} 30$. 
Notation. - Si $X$ est une variété lisse et $Z$ une sous-variété lisse de $X$, on note $B_{Z}(X)$ la variété obtenue en éclatant $X$ le long de $Z$. Rappelons que si $E$ est le diviseur exceptionnel de l'éclatement, $E$ est isomorphe à $\mathbb{P}\left(N_{Z / X}\right)$ où $N_{Z / X}$ est le fibré normal de $Z$ dans $X$. Si $X$ est une variété torique et $Z$ est une sous-variété torique, alors $B_{Z}(X)$ est torique et l'éclatement $\pi: B_{Z}(X) \rightarrow X$ est équivariant.

Notre travail s'articule de la façon suivante : nous rappelons dans un premier temps les notations classiques en géométrie torique et l'énoncé principal de la théorie de Mori des variétés toriques projectives d'après Miles Reid [Rei83]. On en déduit un critère de projectivité pour les variétés toriques qui sont projectives après un éclatement. Dans les deuxième et troisième parties, si $X$ est une variété torique non projective contenant une courbe $C$ torique de sorte que $\widetilde{X}:=B_{C}(X)$ soit projective, nous classifions les contractions Mori extrémales sur $\widetilde{X}$ dont le lieu exceptionnel rencontre le diviseur exceptionnel de l'éclatement $\widetilde{X} \rightarrow X$. Nous montrons en particulier que toutes sont des éclatements le long de centres lisses dans une variété lisse et que seuls trois phénomènes peuvent se produire dans le cas où $\widetilde{X}$ possède une telle contraction : soit $(X, C)$ possède une réduction triviale, soit $X$ est obtenue via un flip interdit d'une variété torique projective, soit $X$ est obtenue via une transformation élémentaire (dite «de Maruyama») d'une variété torique projective, ces termes étant introduits et illustrés au $\S 2$. Nous donnons aussi deux conséquences pour les variétés toriques non projectives devenant de Fano après éclatement le long d'une courbe, en montrant en particulier que ceci ne se produit pas en dimension 3. Ce dernier résultat n'utilise pas la classification des variétés toriques de Fano de dimension 3. Dans la dernière partie, nous reprenons et détaillons une construction d'Ewald, permettant de donner des exemples de variétés toriques non projectives pour toutes les situations étudiées précédemment, ces variétés possédant de plus un petit groupe de Picard.

Remerciements. - À Michel Brion et Laurent Manivel pour de nombreuses discussions et leurs lectures critiques de versions préliminaires, à Alexis Marin qui m'a permis d'interpréter géométriquement la construction d'Ewald et à Chris Peters qui m'a indiqué les travaux de Maruyama.

\section{Préliminaires de géométrie torique}

1.1. Premières définitions et notations. - Les références usuelles de géométrie torique sont [Ewa96], [Fu193] et [Oda88].

Si $M$ est un réseau de rang $n$, une variété torique $X_{\Delta}$ de dimension $n$ est définie par la donnée d'un éventail $\Delta$, subdivision de l'espace vectoriel dual $N_{\mathbb{Q}}:=\operatorname{Hom}(M, \mathbb{Z}) \otimes_{\mathbb{Z}} \mathbb{Q}$ par des cônes rationnels polyédraux. Rappelons qu'il

TOME $128-2000-\mathrm{N}^{\circ} 3$ 
y a une correspondance bijective entre les orbites $\left(\mathrm{du}\right.$ tore $\left.\operatorname{Hom}\left(M, \mathbb{C}^{*}\right)\right)$ de codimension $r$ dans $X_{\Delta}$ et les cônes de dimension $r$ de $\Delta$. Comme annoncé dans l'introduction, toutes les variétés toriques $X_{\Delta}$ seront supposées lisses (ce qui signifie que tous les cônes maximaux de $\Delta$ sont simpliciaux, engendrés par une base de $N:=\operatorname{Hom}(M, \mathbb{Z})$ ) et complètes (ce qui signifie que le support de $\Delta$ est $\left.N_{\mathbb{Q}}\right)$.

Les générateurs dans $N$ des faces de dimension 1 de $\Delta$ seront notés $e_{1}, \ldots, e_{\rho+n}$; ils correspondent aux diviseurs irréductibles invariants de $X_{\Delta}$ et $\rho$ est le nombre de Picard de $X_{\Delta}$.

1.2. Éclatements le long de courbes toriques. - Soient $X$ une variété torique d'éventail $\Delta$ et $C$ une courbe torique de $X$. Quitte à renuméroter les $e_{i}$, $C$ est donnée par le cône de dimension $n-1$ (appelé aussi «mur $\rangle)\left\langle e_{1}, \ldots, e_{n-1}\right\rangle$. Ce mur est face de deux cônes maximaux $\left\langle e_{1}, \ldots, e_{n-1}, e_{n}\right\rangle$ et $\left\langle e_{1}, \ldots, e_{n-1}, e_{n+1}\right\rangle$ correspondant aux deux points fixes du tore sur $C$.

La variété $\widetilde{X}=B_{C}(X)$ est définie par l'éventail $\widetilde{\Delta}$ construit de la façon suivante : on note $e=e_{1}+\cdots+e_{n-1}$, on enlève à $\Delta$ les deux cônes maximaux $\left\langle e_{1}, \ldots, e_{n-1}, e_{n}\right\rangle$ et $\left\langle e_{1}, \ldots, e_{n-1}, e_{n+1}\right\rangle$ (ainsi que leurs faces) et on les remplace par les $2 n-2$ cônes maximaux de la forme

$$
\left\langle e_{1}, \ldots, \widehat{e_{j}}, \ldots, e_{n-1}, e, e_{n}\right\rangle \text { et }\left\langle e_{1}, \ldots, \widehat{e_{j}}, \ldots, e_{n-1}, e, e_{n+1}\right\rangle, \quad 1 \leq j \leq n-1
$$

(avec toutes leurs faces) (la notation $\widehat{e_{j}}$ signifie qu'on enlève $e_{j}$ ) - voir la figure 1.

Les courbes toriques irréductibles sur le diviseur exceptionnel $E$ de l'éclatement $\pi: \widetilde{X} \rightarrow X$ sont les $n-1$ courbes $\widetilde{C}_{j}:=\left\langle e_{1}, \ldots, \widehat{e_{j}}, \ldots, e_{n-1}, e\right\rangle$, $1 \leq j \leq n-1$ qui se projettent isomorphiquement par $\pi \operatorname{sur} C$ et pour $1 \leq i<j \leq n-1$ les courbes

$$
\left\langle e_{1}, \ldots, \widehat{e_{i}}, \ldots, \widehat{e_{j}}, \ldots, e_{n-1}, e, e_{n}\right\rangle \quad \text { et } \quad\left\langle e_{1}, \ldots, \widehat{e_{i}}, \ldots, \widehat{e_{j}}, \ldots, e_{n-1}, e, e_{n+1}\right\rangle
$$

contractées par $\pi$.

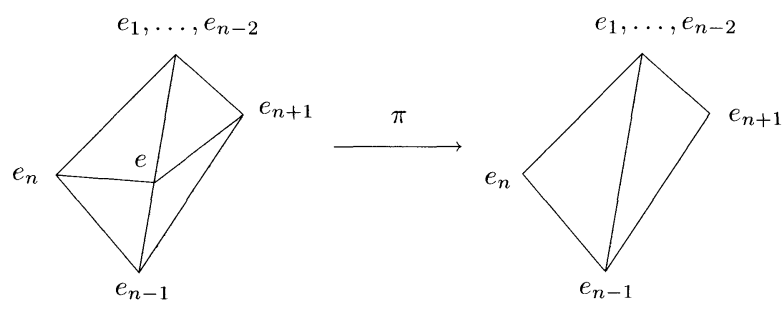

Figure 1

Remarque. - Il y a une description analogue en terme d'éventails des éclatements le long de centres toriques de dimension supérieure à 1 ( $c f$. [Oda88, p. 38]). 
Convention. - La trace sur la sphère unité $S^{n-1}$ d'un éventail $\Delta$ de $\mathbb{R}^{n}$ définit un découpage de $S^{n-1}$. Toutes les figures de ce texte représentent la trace sur la sphère $S^{n-1}$ d'une partie d'un éventail $n$-dimensionnel. Si la figure représente une application torique entre deux variétés toriques, les parties non représentées des éventails ne sont pas modifiées par l'application.

1.3. Théorie de Mori torique, d'après Miles Reid. - Si $X$ est une variété projective, on note

$$
\mathrm{N}_{1}(X)=\left\{\sum_{i} a_{i} C_{i} \mid a_{i} \in \mathbb{Q}, C_{i} \text { courbe irréductible de } X\right\} / \equiv
$$

où $\equiv$ désigne l'équivalence numérique. Le cône de Mori, ou cône des courbes effectives, est le sous-cône de $\mathrm{N}_{1}(X)$ défini par

$$
\mathrm{NE}(X)=\left\{Z \in \mathrm{N}_{1}(X) \mid Z \equiv \sum_{i} a_{i} C_{i}, a_{i} \geq 0\right\}
$$

Si $X$ est torique, alors (voir [Rei83]) $\mathrm{NE}(X)$ est polyédral, engendré par les classes des courbes invariantes. De plus, pour toute arête $R$ de $\mathrm{NE}(X)$, il y a une contraction $\varphi_{R}: X \rightarrow Y$ de $X$ sur une variété torique projective éventuellement singulière $Y$ telle que les courbes irréductibles contractées par $\varphi_{R}$ sont exactement celles dont la classe dans $\mathrm{N}_{1}(X)$ appartient à $R$.

Convention. - Pour une arête $R$ de $\mathrm{NE}(X)$, nous dirons que la contraction extrémale $\varphi_{R}$ est Mori extrémale si et seulement si $-K_{X} \cdot R>0$.

1.3.1. Comportement du cône de Mori. — Soient $\varphi_{R}: X \rightarrow Y$ une contraction extrémale et

$$
\left(\varphi_{R}\right)_{*}: \mathrm{N}_{1}(X) \longrightarrow \mathrm{N}_{1}(Y)=\mathrm{N}_{1}(X) /(R+(-R))
$$

la projection induite par $\varphi_{R}$. Alors, le cône de Mori $\mathrm{NE}(Y)$ est égal à $\left(\varphi_{R}\right)_{*}(\mathrm{NE}(X))$. En particulier, les arêtes de $\mathrm{NE}(Y)$ sont images d'arêtes de $\mathrm{NE}(X)$.

1.3.2. Description des contractions extrémales. - Soit $\omega$ une courbe invariante extrémale (i.e. $R:=\mathbb{Q}^{+}[\omega]$ est une arête de $\mathrm{NE}(X)$ ) que l'on peut supposer, quitte à renuméroter les $e_{i}$, définie par le «mur» $\left\langle e_{1}, \ldots, e_{n-1}\right\rangle$, face de deux cônes maximaux

$$
\left\langle e_{1}, \ldots, e_{n-1}, e_{n}\right\rangle \text { et }\left\langle e_{1}, \ldots, e_{n-1}, e_{n+1}\right\rangle \text {. }
$$

TOME $128-2000-\mathrm{N}^{\circ} 3$ 
Comme $X$ est lisse, $e_{1}, \ldots, e_{n}$ forment une base du réseau et il y a donc une unique relation

$$
e_{n+1}+e_{n}+\sum_{i=1}^{n-1} a_{i} e_{i}=0
$$

où les $a_{i}$ sont des entiers. Rappelons ici que le fibré normal de $\omega$ dans $X$ est isomorphe à $\bigoplus_{i=1}^{n-1} \mathcal{O}_{\mathbb{P}^{1}}\left(a_{i}\right)$, ceci étant vrai même si $\omega$ n'est pas extrémale.

On note :

$$
\begin{aligned}
& \alpha=\operatorname{card}\left\{i \in[1, \ldots, n-1] \mid a_{i}<0\right\}, \\
& \beta=\operatorname{card}\left\{i \in[1, \ldots, n-1] \mid a_{i} \leq 0\right\} .
\end{aligned}
$$

Avec ces notations, $\varphi_{R}$ est birationnelle si et seulement si $\alpha \neq 0$; le lieu exceptionnel $A(R)$ de $\varphi_{R}$ dans $X$ est alors de dimension $n-\alpha$, son image $B(R)=\varphi_{R}(A(R))$ dans $Y$ est de dimension $\beta-\alpha$, et la restriction de $\varphi_{R}$ à $A(R)$ est un morphisme plat à fibres des espaces projectifs à poids de dimension $n-\beta$. Si $\alpha=0, Y$ est projective lisse de dimension $\beta$ et $\varphi_{R}$ est une fibration lisse. (Attention : les coquilles de l'énoncé (2.5) dans [Rei83] sont corrigées dans [Oda88, p. 111]).

1.4. Un critère de projectivité torique. - Nous donnons ici une première application de la théorie de Mori torique.

Lemme 1. - Soit $X$ une variété torique, $Z$ une sous-variété torique de $X$. On suppose que $B_{Z}(X)$ est projective. Alors $X$ est projective si et seulement si toute courbe contenue dans une fibre de l'éclatement $\pi: B_{Z}(X) \rightarrow X$ est extrémale dans $\mathrm{NE}\left(B_{Z}(X)\right)$.

Remarque. - Comme les fibres non triviales de $\pi$ sont des espaces projectifs (lisses), l'arête engendrée par la classe dans $\mathrm{NE}\left(B_{Z}(X)\right)$ d'une courbe contenue dans une fibre de l'éclatement $\pi: B_{Z}(X) \rightarrow X$ est indépendante des choix de la courbe et de la fibre.

Démonstration du lemme. - Le sens direct est un fait général bien connu : soit $F$ une courbe rationnelle contenue dans une fibre non triviale de $\pi$. Si $X$ est projective, soit $H$ un diviseur ample sur $X$. Si $F \equiv C_{1}+C_{2}$ dans $\mathrm{NE}\left(B_{Z}(X)\right)$, alors

$$
0=\pi^{*} H \cdot F=\pi^{*} H \cdot C_{1}+\pi^{*} H \cdot C_{2},
$$

donc

$$
\pi^{*} H \cdot C_{1}=\pi^{*} H \cdot C_{2}=0 .
$$

Ainsi les $C_{i}$ sont contenues dans une fibre de $\pi$, donc numériquement proportionnelles à $F$, autrement dit $F$ est extrémale. Réciproquement, si $F$ est extrémale, 
la contraction extrémale $\varphi$ associée est birationnelle de lieu exceptionnel égal au diviseur exceptionnel de $\pi$ : en effet, la classe de toute courbe incluse dans une fibre de $\pi$ appartient à l'arête $\mathbb{Q}^{+}[F]$, donc toutes les fibres non triviales de $\pi$ sont contractées par $\varphi$, et toute courbe contractée par $\varphi$ est incluse dans $E$ car $E \cdot F<0$. Ainsi, toute fibre de $\pi$ est contenue dans une fibre de $\varphi$, et les fibres de $\varphi$ étant des espaces projectifs à poids, la restriction de $\pi$ à toute fibre de $\varphi$ la contracte sur un point, c'est donc que $\varphi=\pi$ et $X$ est projective.

Remarque. - Cet énoncé est un énoncé torique, faux pour des variétés projectives quelconques (voir [Bon96, § 2.4])!

\section{Trois exemples de contractions extrémales}

Dans cette partie, la situation qui nous intéresse est la suivante : $X$ est une variété torique non projective contenant une courbe $C$ torique de sorte que $\widetilde{X}:=B_{C}(X)$ soit projective. On note $\pi: \widetilde{X} \rightarrow X$ l'éclatement de $X$ le long de $C$ et $E$ le diviseur exceptionnel de $\pi$.

Question. - Que peut-on dire des contractions Mori extrémales sur $\widetilde{X}$ dont le lieu exceptionnel rencontre $E$ ?

Nous donnons trois exemples différents de situations possibles.

2.1. Exemple : "réduction triviale". - Supposons donnée une variété torique non projective $X^{\prime}$, contenant une courbe $C^{\prime}$ torique de sorte que $Y:=B_{C^{\prime}}\left(X^{\prime}\right)$ soit projective; on note $\pi^{\prime}$ l'éclatement $Y \rightarrow X^{\prime}$. Soit alors $X$ la variété torique obtenue en éclatant $X^{\prime}$ en un point fixe $p$ appartenant à $C^{\prime}$. Comme $X^{\prime}$, la variété $X$ est non projective, et la variété $\widetilde{X}:=B_{C}(X)$ où la courbe $C$ est la transformée stricte de $C^{\prime}$ est projective : en effet, $\widetilde{X}$ est la variété obtenue en éclatant $Y$ le long de la sous-variété de codimension $2\left(\pi^{\prime}\right)^{-1}(p)$. D'après le lemme 1 , l'éclatement $\widetilde{X} \rightarrow Y$ est une contraction Mori extrémale $\varphi_{\mathbb{Q}^{+}[\omega]}$ dont le lieu exceptionnel rencontre $E$.

Autrement dit, on a un diagramme commutatif :

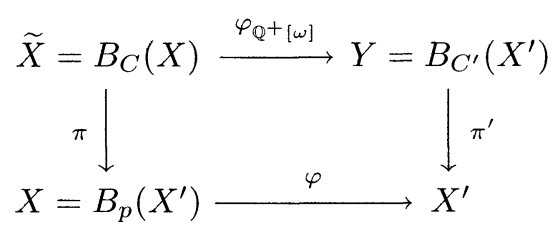

La figure 2 est la version "géométrique» de ce qui précède.

Convention. - Si le couple $(X, C)$ est obtenu par le procédé décrit précédemment, nous dirons que ce couple possède une réduction triviale.

TOME $128-2000-\mathrm{N}^{\circ} 3$ 


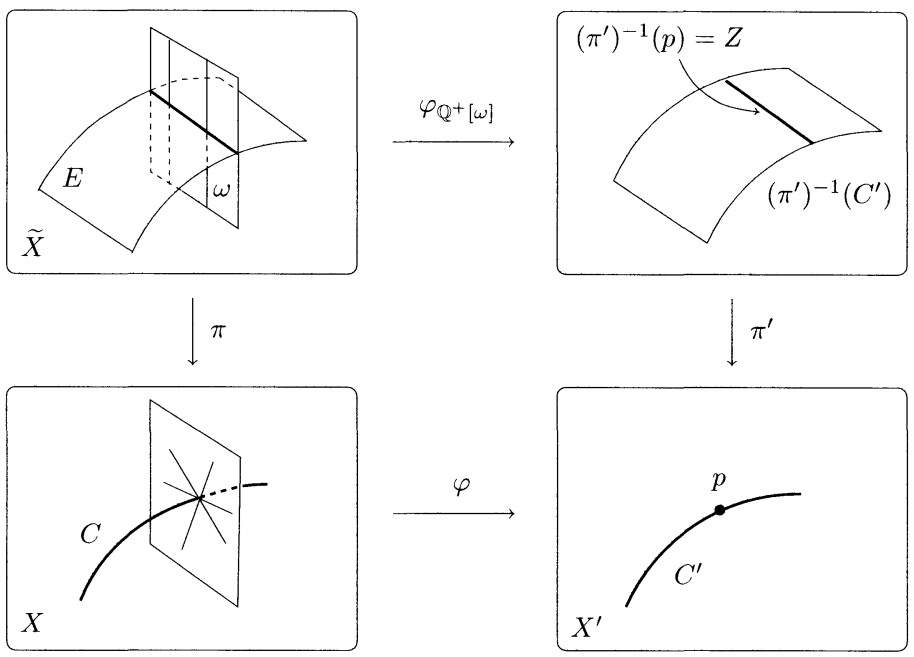

Figure 2. Réduction triviale

2.2. Exemple : "flip interdit". - Démontrons la proposition suivante :

Proposition 1. - Soit $Y$ une variété torique projective de dimension $n$ contenant une sous-variété torique $Z$ vérifiant

$$
\left(Z, N_{Z / Y}\right) \simeq\left(\mathbb{P}^{n-2}, \mathcal{O}_{\mathbb{P}^{n-2}}(-1)^{\oplus 2}\right)
$$

et soit $X$ la variété torique obtenue en éclatant $Y$ le long de $Z$ puis en contractant les $\{*\} \times \mathbb{P}^{n-2}$ du diviseur exceptionnel $E \simeq \mathbb{P}^{1} \times \mathbb{P}^{n-2}$ :

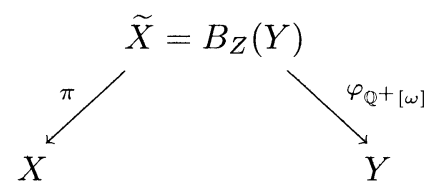

Alors $X$ est projective si et seulement si la classe d'une courbe incluse dans $Z$ est extrémale dans le cône de Mori des courbes effectives de $Y$.

En particulier, si la classe d'une courbe incluse dans $Z$ n'est pas extrémale dans le cône de Mori des courbes effectives de $Y$, la variété $X$ construite est non projective, le devient après éclatement le long d'une courbe $C$ vérifiant

$$
\left(C, N_{C / X}\right) \simeq\left(\mathbb{P}^{1}, \mathcal{O}_{\mathbb{P}^{1}}(-1)^{\oplus n-1}\right),
$$


et $\varphi_{\mathbb{Q}^{+}[\omega]}$ est une contraction Mori extrémale dont le lieu exceptionnel est égal à celui de l'éclatement $\pi: \widetilde{X} \rightarrow X$.

Démonstration de la proposition. - Si la classe d'une courbe incluse dans $Z$ est extrémale, $\pi \circ \varphi_{\mathbb{Q}^{+}[\omega]}^{-1}$ est un flip (resp. un flop) si $n \geq 4$ (resp. si $n=3$ ) et $X$ est projective. Réciproquement, soit $\bar{F}$ une courbe rationnelle incluse dans $Z$, non extrémale dans le cône de Mori des courbes effectives de $Y:[\bar{F}]=\left[C_{1}\right]+\left[C_{2}\right]$, où les $\left[C_{i}\right]$ sont effectives, non incluses dans $Z$. Si $C_{i}^{\prime}$ désigne la transformée stricte de $C_{i}$ pour $\bar{\pi}$, et si $F$ est une courbe de $\widetilde{X}$, incluse dans une fibre de $\pi$ telle que $\bar{\pi}_{*} F=\bar{F}$, on a

$$
[F]=\left[C_{1}^{\prime}\right]+\left[C_{2}^{\prime}\right]+a\left[C^{\prime}\right]
$$

où $a$ est un entier et $\left[C^{\prime}\right]$ la classe d'une fibre non triviale de $\bar{\pi}$. Or

$$
-1=E \cdot F=E \cdot C_{1}^{\prime}+E \cdot C_{2}^{\prime}+a E \cdot C^{\prime}=E \cdot C_{1}^{\prime}+E \cdot C_{2}^{\prime}-a
$$

d'où

$$
a=1+E \cdot C_{1}^{\prime}+E \cdot C_{2}^{\prime} \geq 1
$$

car $E \cdot C_{i}^{\prime} \geq 0$. Ainsi, la classe de $[F]$ n'est pas extrémale dans le cône de Mori des courbes effectives de $\tilde{X}$, donc $X$ n'est pas projective d'après le lemme 1 .

Convention. - Si une variété $X$ non projective est obtenue par le procédé décrit précédemment, nous dirons que cette variété est obtenue via un flip interdit d'une variété projective : comme mentionné précédemment, à partir de la dimension 4, cette transformation de $Y$ vers $X$ est un flip si la classe d'une courbe incluse dans $Z$ est extrémale dans le cône de Mori des courbes effectives de $Y$.

2.3. Exemple : "transformation élémentaire". - Soit $Y$ une variété torique projective de dimension $n$ contenant un diviseur torique $\bar{E}$. Supposons que $\bar{E}$ soit isomorphe à $\mathbb{P}(\mathcal{E})$ où $\mathcal{E}$ est un fibré vectoriel de rang $n-1$ sur $\mathbb{P}^{1}$ et notons $\lambda: \bar{E}=\mathbb{P}(\mathcal{E}) \rightarrow \mathbb{P}^{1}$ la projection. Supposons que le fibré normal de $\bar{E}$ dans $Y$ soit de la forme $\lambda^{*} \mathcal{O}_{\mathbb{P}^{1}}(a)$ (autrement dit, il est trivial en restriction aux fibres de $\lambda$ ) et soit $\mathcal{E}^{\prime}$ un sous-fibré de $\mathcal{E}$ de rang $n-2$ de sorte que la sous-variété $Z=\mathbb{P}\left(\mathcal{E}^{\prime}\right)$ soit torique. Soit $\widetilde{X}=B_{Z}(Y)$ la variété torique obtenue en éclatant $Y$ le long de $Z$. D'après le lemme 1 , l'éclatement $\widetilde{X} \rightarrow Y$ est une contraction Mori extrémale $\varphi_{\mathbb{Q}^{+}[\omega]}$. De plus, la transformée stricte $E$ de $\bar{E}$ est isomorphe à $\bar{E}$ (en particulier, $\lambda$ induit une fibration $\tilde{\lambda}: E \rightarrow \mathbb{P}^{1}$ ) et son fibré normal $N_{E / \widetilde{X}}$ est égal à $\mathcal{O}_{E}(-1) \otimes \tilde{\lambda}^{*} \mathcal{O}_{\mathbb{P}^{1}}(a)$. Il existe donc une variété $X$ torique contenant une courbe torique $C \simeq \mathbb{P}^{1}$ de sorte que $\widetilde{X}=B_{C}(X)$ et que le diviseur exceptionnel de l'éclatement $\pi: \widetilde{X} \rightarrow \mathbb{P}^{1}$ soit égal à $E$ avec $\pi_{\mid E}=\tilde{\lambda}$. Par construction, $\varphi_{\mathbb{Q}^{+}}[\omega]$ est une contraction Mori extrémale dont le lieu exceptionnel $F$ rencontre $E$.

TOME $128-2000-\mathrm{N}^{\circ} 3$ 
Autrement dit, on a un diagramme commutatif :

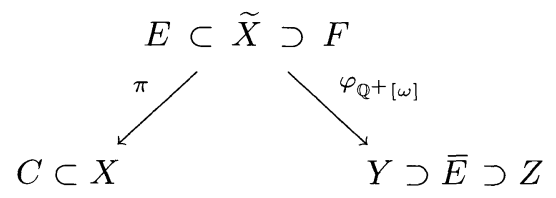

La figure 3 est la version «géométrique» de ce qui précède.

Convention. - Si une variété $X$ est obtenue par le procédé décrit précédemment, nous dirons que cette variété est obtenue via une transformation élémentaire d'une variété projective.

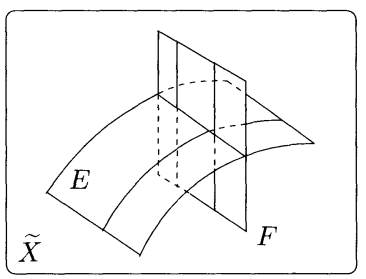

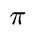

$\pi$
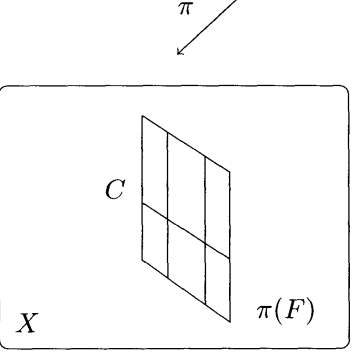

$\varphi \mathbb{Q}^{+}[\omega]$

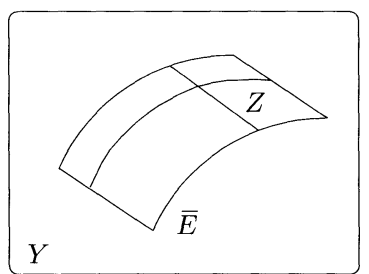

Figure 3. Transformation élémentaire.

Lien avec les transformations élémentaires de Maruyama. - Notons $S$ l'espace total du fibré $\mathcal{O}_{\mathbb{P}^{1}}(a), q: S \rightarrow \mathbb{P}^{1}$ la projection et $U$ le produit fibré (torique) de $\lambda$ et $q$ au-dessus de $\mathbb{P}^{1}$ :

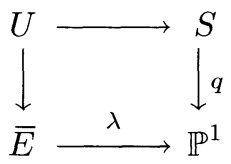

L'hypothèse faite sur $N_{\bar{E} / Y}$ implique l'existence d'un voisinage torique $V$ de $\bar{E}$ dans $Y$ isomorphe à $U=\mathbb{P}\left(q^{*} \mathcal{E}\right)$. Enfin, si $T$ désigne la section nulle de $q$ dans $S$ 
et si $\widetilde{V}=\left(\varphi_{\mathbb{Q}^{+}[\omega]}\right)^{-1}(V)$, alors le diagramme

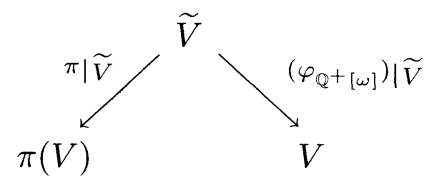

est une transformation élémentaire de Maruyama [Mar82, §1].

À la lumière de ce qui précède, nous pouvons dire que l'exemple 2.3 est une «compactification équivariante d'une transformation élémentaire torique ».

\section{Classification de certaines contractions Mori extrémales}

Dans toute cette partie, $X$ est une variété torique non projective de dimension $n$, d'éventail $\Delta$ contenant une courbe $C$ torique de sorte que $\widetilde{X}:=B_{C}(X)$ soit projective, d'éventail $\widetilde{\Delta}$. On note $\pi: \widetilde{X} \rightarrow X$ l'éclatement de $X$ le long de $C$, $E$ le diviseur exceptionnel de $\pi$. Quitte à renuméroter les faces de dimension 1 de $\Delta$, on suppose que $C$ est donnée par un cône $\left\langle e_{1}, \ldots, e_{n-1}\right\rangle$ de dimension $n-1$, face de deux cônes maximaux $\left\langle e_{1}, \ldots, e_{n-1}, e_{n}\right\rangle$ et $\left\langle e_{1}, \ldots, e_{n-1}, e_{n+1}\right\rangle$.

Le but de cette partie est de classifier les contractions Mori extrémales de $\widetilde{X}$ dont le lieu exceptionnel rencontre $E$. Nous y démontrons le résultat suivant :

ThÉORÈme 1. - Soit $X$ une variété torique non projective contenant une courbe $C$ torique de sorte que $\widetilde{X}:=B_{C}(X)$ soit projective, soit $\omega$ dans $\widetilde{X}$ une courbe Mori extrémale rencontrant le diviseur exceptionnel $E$ et $\varphi_{\mathbb{Q}^{+}[\omega]}: \widetilde{X} \rightarrow Y$ la contraction extrémale associée. Alors :

- soit le couple $(X, C)$ possède une réduction triviale;

- soit $X$ est obtenue via un flip interdit d'une variété torique projective;

- soit $X$ est obtenue via une transformation élémentaire d'une variété torique projective.

Dans tous les cas, la contraction extrémale $\varphi_{\mathbb{Q}^{+}[\omega]}$ est de la forme décrite dans les exemples $2.1,2.2$ et 2.3 respectivement.

Commentaires.

(i) Dans tous les cas, l'image $Y$ de la contraction extrémale $\varphi_{\mathbb{Q}^{+}[\omega]}$ est lisse et $\varphi_{\mathbb{Q}^{+}[\omega]}$ est l'éclatement de $Y$ le long d'un centre lisse; ce phénomène est remarquable.

(ii) Dans le deuxième cas, $E \cdot \omega=-1$, dans les deux autres $E \cdot \omega=1$.

(iii) Tous ces exemples se produisent en toutes dimensions supérieures à 3 , nous donnerons des constructions explicites dans la partie 4 avec «petits» groupes de Picard.

$$
\text { TOME } 128-2000-\mathrm{N}^{\circ} 3
$$


(iv) Cet énoncé n'est pertinent que s'il existe une courbe Mori extrémale rencontrant $E$, hypothèse que nous ne savons pas vérifier en général. Dans le corollaire suivant, cette hypothèse est vérifiée.

Corollaire 1. - Soit $X$ une variété torique non projective contenant une courbe $C$ torique de sorte que $\tilde{X}:=B_{C}(X)$ soit une variété de Fano. Alors $X$ est obtenue via un fip interdit d'une variété torique projective.

Démonstration du corollaire. - Soit $C$ une courbe contenue dans une fibre de l'éclatement. Alors,

$$
[C] \equiv \sum_{i=1}^{p} a_{i}\left[C_{i}\right]
$$

où les $C_{i}$ sont des courbes Mori extrémales et les $a_{i}$ des rationnels positifs. Si $E$ est le diviseur exceptionnel de l'éclatement, comme $E \cdot C<0$, l'une au moins des $C_{i}$ vérifie $E \cdot C_{i}<0$. Cette courbe satisfait donc les hypothèses du théorème, et comme $E \cdot C_{i}<0$, la contraction extrémale associée est un flip interdit.

En dimension 3, on en déduit :

Corollaire 2. - Soit $X$ une variété torique de dimension 3 contenant une courbe $C$ torique de sorte que $\widetilde{X}:=B_{C}(X)$ soit une variété de Fano. Alors $X$ est projective.

Mentionnons que cet énoncé est faux hors du contexte torique : il y a des variétés analytiques complexes non projectives de dimension 3 devenant de Fano après éclatement le long d'une courbe.

Démonstration du corollaire. - Si $X$ n'est pas projective, le corollaire 1 affirme que $X$ est obtenue via un flip interdit d'une variété torique projective. Comme $-K_{\widetilde{X}}$ est ample, $-K_{X}$ est d'intersection strictement positive avec toutes les courbes de $X$ distinctes de $C$. Or, comme

$$
N_{C / X}=\mathcal{O}_{\mathbb{P}^{1}}(-1)^{\oplus 2}
$$

on a $-K_{X} \cdot C=0$. Mais alors, si $A$ est un diviseur nef dans une variété torique, d'intersection strictement positive avec toutes les courbes toriques à l'exception d'une seule notée $C$, et si $D$ est un diviseur lisse vérifiant $D \cdot C=1$, le diviseur $A+\varepsilon D$ est un $\mathbb{Q}$-diviseur strictement positif avec toutes les courbes toriques, donc ample, dès que $\varepsilon$ est un rationnel strictement positif assez petit. Ainsi, $X$ est projective.

Remarque. - Le corollaire 2 découle aussi de la classification des variétés toriques de Fano de dimension 3 (voir [Bat82], [WWa82]). En effet, cette dernière 
montre que $\rho(\tilde{X}) \leq 5$, donc $\rho(X) \leq 4$. Or il n'y a qu'une seule variété torique non projective de dimension 3 dont le rang du groupe de Picard est inférieur ou égal à 4 (celle dont l'éventail est rappelé au $§ 4.1 .1$ de ce texte) [Oda88] et il est facile de voir que cette dernière ne devient pas de Fano après éclatement le long d'une courbe. La preuve du corollaire 2 évite d'utiliser cette classification.

Le reste de cette partie est consacré à la démonstration du théorème 1 .

Supposons donc l'existence d'une courbe torique $\omega$ de $\tilde{X}:=B_{C}(X)$ Mori extrémale vérifiant $E \cap \omega \neq \emptyset$. L'analyse distingue les cas $E \cdot \omega \geq 0$ et $E \cdot \omega<0$.

\subsection{Le cas où $\boldsymbol{E} \cdot \boldsymbol{\omega} \geq \mathbf{0}$.}

\subsubsection{Quelques réductions.}

Lemme 2. - Sous les hypothèses précédentes, la courbe $\omega$ n'est pas incluse dans $E$.

Démonstration. - Supposons au contraire que $\omega \subset E$. Comme $E \cdot \omega \geq 0$, $\omega$ n'est pas incluse dans une fibre de $\pi$, donc $\pi_{\mid \omega}: \omega \rightarrow C$ est un isomorphisme d'après 1.2. De là,

$$
K_{X} \cdot C=\pi^{*} K_{X} \cdot \omega=\left(K_{\widetilde{X}}-(n-2) E\right) \cdot \omega
$$

et donc

$$
\chi\left(N_{C / X}\right)=-K_{X} \cdot C+n-3=\left(-K_{\widetilde{X}}+(n-2) E\right) \cdot \omega+n-3>0 .
$$

Montrons maintenant que ceci entraîne que $X$ est projective. En effet l'égalité précédente montre que $C$ n'est pas rigide et la non-projectivité de $X$ contredirait le lemme suivant :

Lemme 3. - Soit $X$ une variété torique, $C$ une courbe torique de $X$. On suppose que $B_{C}(X)$ est projective et que $C$ se déforme dans $X$. Alors $X$ est projective.

Remarque. - Dans cet énoncé, les déformations de $C$ sont nécessairement non toriques.

Démonstration du lemme. - Comme $X$ est torique, un diviseur sur $X$ est ample si et seulement si son intersection avec toute courbe effective est strictement positive. De là, si $H$ est un diviseur ample sur $B_{C}(X)$, et si $\pi: B_{C}(X) \rightarrow X$ est l'éclatement, le diviseur $H^{\prime}:=\pi(H)$ est ample sur $X:$ en effet, si $\omega$ est une courbe irréductible de $X$ distincte de $C$, alors $H^{\prime} \cdot \omega \geq H \cdot \widetilde{\omega}>0$ où $\widetilde{\omega}$ est la transformée stricte de $\omega$. Comme $C$ se déforme en une courbe $\omega_{C}$, on a $H^{\prime} \cdot C=H^{\prime} \cdot \omega_{C}>0$ d'après ce qui précède. Ainsi, $H^{\prime}$ est ample sur $X$. 
Ainsi, $\omega$ est «transverse》 à $E$ et quitte à permuter les $e_{1}, \ldots, e_{n-1}$, on peut supposer que $\omega=\left\langle e_{1}, \ldots, e_{n-2}, e_{n}\right\rangle$ (et donc $\left.E \cdot \omega=1\right)$. Le mur $\left\langle e_{1}, \ldots, e_{n-2}, e_{n}\right\rangle$ définissant $\omega$ est face de deux cônes maximaux

$$
\left\langle e_{1}, \ldots, e_{n-2}, e_{n}, e\right\rangle \quad \text { et }\left\langle e_{1}, \ldots, e_{n-2}, e_{n}, e^{\prime}\right\rangle
$$

pour un certain $e^{\prime}$, générateur entier d'une face de dimension 1 de $\widetilde{\Delta}$. Remarquons que $e^{\prime}$ peut être égal à $e_{n+1}$ et est distinct de $e_{n-1}$ - voir la figure 4 .

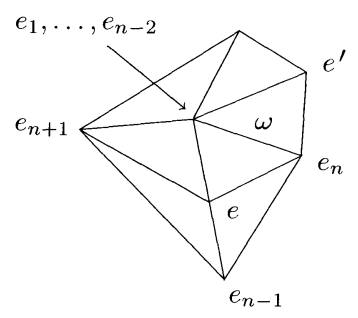

Figure 4

Il y a alors une relation

$$
e^{\prime}+e+\sum_{i=1}^{n-2} b_{i} e_{i}+b_{n} e_{n}=0
$$

où les $b_{i}$ et $b_{n}$ sont des entiers.

Lemme 4. - Les entiers $b_{i}$ sont négatifs ou nuls pour tout $i=1, \ldots, n-2, n$.

Démonstration. - Sinon, on a $b_{i}>0$ pour un certain $i$, et d'après Reid [Rei83, cor. 2.10 (i)], les deux courbes

$$
\omega^{\prime}:=\left\langle e_{1}, \ldots, \widehat{e_{i}}, \ldots, e_{n-2}, e_{n}, e\right\rangle \quad \text { et } \quad \omega^{\prime \prime}:=\left\langle e_{1}, \ldots, \widehat{e_{i}}, \ldots, e_{n-2}, e_{n}, e^{\prime}\right\rangle
$$

(resp. $\left\langle e_{1}, \ldots, e_{n-2}, e\right\rangle$ et $\left.\left\langle e_{1}, \ldots, e_{n-2}, e^{\prime}\right\rangle\right)$ si $i \leq n-2$ (resp. si $i=n$ ) sont sur l'arête $\mathbb{Q}^{+}[\omega]$.

Mais alors $\omega^{\prime}:=\left\langle e_{1}, \ldots, \widehat{e_{i}}, \ldots, e_{n-2}, e_{n}, e\right\rangle$ (resp. $\left\langle e_{1}, \ldots, e_{n-2}, e\right\rangle$ ) est une courbe Mori extrémale incluse dans $E$ et d'intersection positive avec $E$, ce qui n'est pas possible d'après le lemme 2 .

Comme la quantité

$$
-K_{\widetilde{X}} \cdot \omega=2+\sum_{i=1}^{n-2} b_{i}+b_{n}
$$

BULLETIN DE LA SOCIÉTÉ MATHÉMATIQUE DE FRANCE 
est strictement positive, le lemme précédent et la description des contractions extrémales rappelées en 1.3 impliquent que seules deux possibilités ont lieu $a$ priori.

a) Tous les entiers $b_{i}, 1 \leq i \leq n-2$ et $b_{n}$ sont nuls et dans ce cas la contraction extrémale $\varphi_{\mathbb{Q}^{+}[\omega]}$ est une fibration $\varphi_{\mathbb{Q}^{+}[\omega]}: \widetilde{X} \rightarrow Y$ de fibres isomorphes à $\mathbb{P}^{1}$.

b) Un et un seul des entiers $b_{i}$ et $b_{n}$ vaut -1 , les autres étant nuls et dans ce cas la contraction extrémale $\varphi_{\mathbb{Q}^{+}[\omega]}$ est birationnelle divisorielle, ses fibres non triviales étant isomorphes à $\mathbb{P}^{1}$.

Montrons que le cas a) est impossible $:$ si $\varphi_{\mathbb{Q}^{+}[\omega]}: \widetilde{X} \rightarrow Y$ est une fibration, ses fibres ont une intersection égale à 1 avec le diviseur $E$ et donc $E$ est isomorphe à $Y$. On en déduit que le groupe de Picard de $Y$ est $\mathbb{Z}^{2}$, donc celui de $\widetilde{X}$ est $\mathbb{Z}^{3}$, donc celui de $X$ est $\mathbb{Z}^{2}$. Mais alors $X$ est projective d'après la classification [Kle88].

3.1.2. Analyse du cas b). - Deux situations différentes peuvent se produire:

(i) $b_{i}=0$ pour tout $1 \leq i \leq n-2$ et $b_{n}=-1$, on a alors $e^{\prime}+e-e_{n}=0$;

(ii) l'un des $b_{i}, 1 \leq i \leq n-2$ est non nul, on peut supposer que $b_{1}=-1$ et on a alors $e^{\prime}+e-e_{1}=0$.

Commençons par traiter la situation (i) : nous montrons que $(X, C)$ possède une réduction triviale.

D'après [Rei83, cor. 2.10 (ii)], pour tout $i$ fixé, $1 \leq i \leq n-2$, l'étoile du cône $n-2$ dimensionnel $\left\langle e_{1}, \ldots, \widehat{e_{i}}, \ldots, e_{n-2}, e_{n}\right\rangle$ est constituée, pour un certain $\nu_{i}$, des quatre cônes maximaux

$$
\begin{array}{cl}
\left\langle e_{1}, \ldots, e_{i}, \ldots, e_{n-2}, e_{n}, e\right\rangle, & \left\langle e_{1}, \ldots, e_{i}, \ldots, e_{n-2}, e_{n}, e^{\prime}\right\rangle \\
\left\langle e_{1}, \ldots, \widehat{e}_{i}, \ldots, e_{n-2}, e_{n}, e, \nu_{i}\right\rangle, & \left\langle e_{1}, \ldots, \widehat{e}_{i}, \ldots, e_{n-2}, e_{n}, e^{\prime}, \nu_{i}\right\rangle .
\end{array}
$$

Or, le mur $\left\langle e_{1}, \ldots, \widehat{e_{i}}, \ldots, e_{n-2}, e_{n}, e\right\rangle$ est déjà face des deux cônes maximaux

$$
\left\langle e_{1}, \ldots, \widehat{e_{i}}, \ldots, e_{n-2}, e_{n}, e, e_{n-1}\right\rangle \text { et }\left\langle e_{1}, \ldots, e_{i}, \ldots, e_{n-2}, e_{n}, e\right\rangle .
$$

C'est donc que $\nu_{i}=e_{n-1}$ pour tout $1 \leq i \leq n-2$. On en déduit que l'étoile de $e_{n}$ est constituée des $2 n-2$ cônes maximaux suivants :

$$
\left\langle e_{1}, \ldots, \widehat{e_{i}}, \ldots, e_{n-1}, e, e_{n}\right\rangle \quad \text { et } \quad\left\langle e_{1}, \ldots, \widehat{e_{i}}, \ldots, e_{n-1}, e^{\prime}, e_{n}\right\rangle, \quad 1 \leq i \leq n-1
$$

Il résulte du raisonnement précédent - voir la figure 5 - que la contraction $\varphi_{\mathbb{Q}^{+}[\omega]}$ envoie $\widetilde{X}$ sur la variété projective lisse $Y$ dont l'éventail est obtenu en remplaçant dans $\widetilde{\Delta}$ l'étoile de $e_{n}$ par la réunion des cônes maximaux $\left\langle e_{1}, \ldots, \widehat{e}_{i}, e_{n-1}, e^{\prime}, e\right\rangle, 1 \leq i \leq n-1$ et leurs faces. 


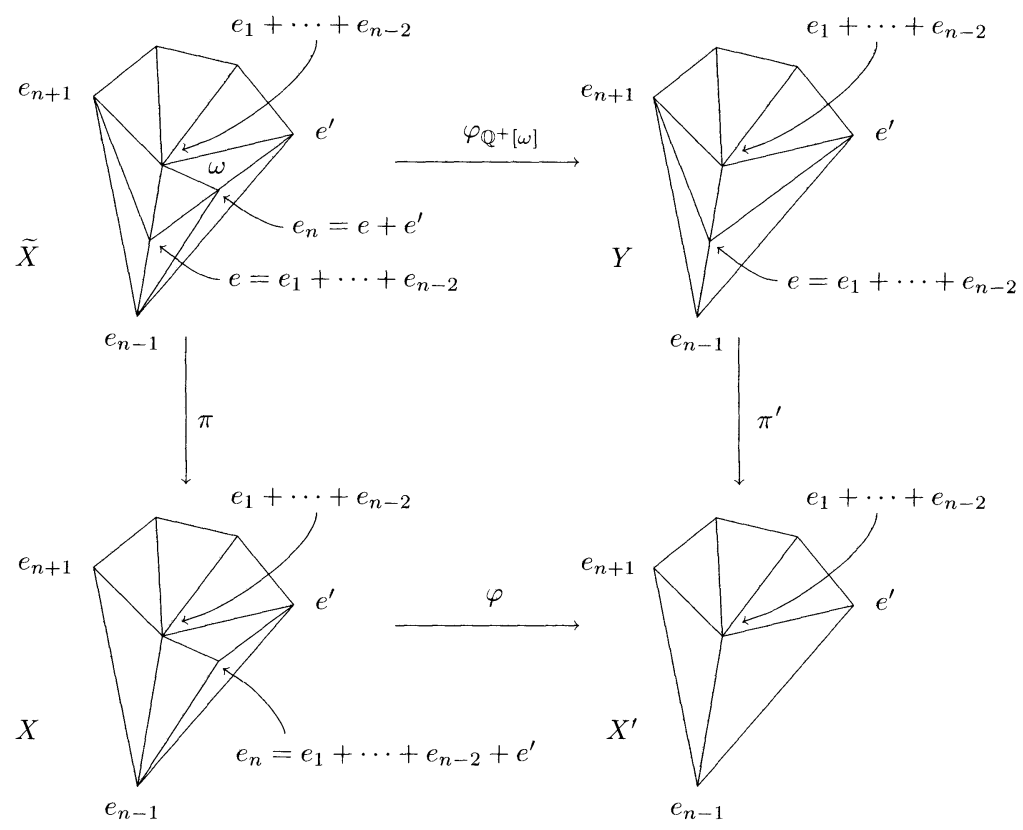

Figure 5. Réduction triviale

Autrement dit, $\varphi_{\mathbb{Q}^{+}[\omega]}: \widetilde{X} \rightarrow Y$ est l'éclatement de $Y$ le long de la sous-variété torique de codimension 2 définie par le cône $\left\langle e, e^{\prime}\right\rangle$.

De même, la relation

$$
e_{n}=e_{1}+\cdots+e_{n-1}+e^{\prime}
$$

montre qu'il existe une variété $X^{\prime}$ non projective lisse telle que $X=B_{p}\left(X^{\prime}\right)$ où $p$ est un point fixe dans $X^{\prime}$ définie par le cône maximal $\left\langle e_{1}, \ldots, e_{n-1}, e^{\prime}\right\rangle$. Enfin, $Y=B_{C^{\prime}}\left(X^{\prime}\right)$ où $C^{\prime}$ est la courbe de $X^{\prime}$ définie par le mur $\left\langle e_{1}, \ldots, e_{n-1}\right\rangle$. Ce qui précède montre que $(X, C)$ possède une réduction triviale.

Traitons la situation (ii) : nous montrons que $X$ est obtenue via une transformation élémentaire d'une variété torique projective

Dans ce cas, on a $e_{1}=e+e^{\prime}$, soit encore $e^{\prime}=-e_{2}-\cdots-e_{n-1}$. Un raisonnement analogue au précédent montre que l'étoile de $e_{1}$ est constituée des $4 n-8$ cônes maximaux suivants :

$$
\begin{array}{cc}
\left\langle e_{1}, \ldots, \widehat{e}_{i}, \ldots, e_{n-1}, e, e_{n}\right\rangle, & \left\langle e_{1}, \ldots, \widehat{e}_{i}, \ldots, e_{n-1}, e, e_{n+1}\right\rangle \\
\left\langle e_{1}, \ldots, \hat{e}_{i}, \ldots, e_{n-1}, e^{\prime}, e_{n}\right\rangle, & \left\langle e_{1}, \ldots, \widehat{e}_{i}, \ldots, e_{n-1}, e^{\prime}, e_{n+1}\right\rangle
\end{array}
$$

pour $2 \leq i \leq n-1$. 
Il en résulte - voir la figure 6 - que la contraction $\varphi_{\mathbb{Q}^{+}}[\omega]$ envoie $\widetilde{X}$ sur la variété projective lisse $Y$ dont l'éventail est obtenu en remplaçant dans $\widetilde{\Delta}$ l'étoile de $e_{1}$ par la réunion des cônes maximaux

$$
\left\langle e_{2}, \ldots, \widehat{e}_{i}, e_{n-1}, e^{\prime}, e, e_{n}\right\rangle, \quad\left\langle e_{2}, \ldots, \widehat{e}_{i}, e_{n-1}, e^{\prime}, e, e_{n+1}\right\rangle, \quad 2 \leq i \leq n-1
$$

et leurs faces.

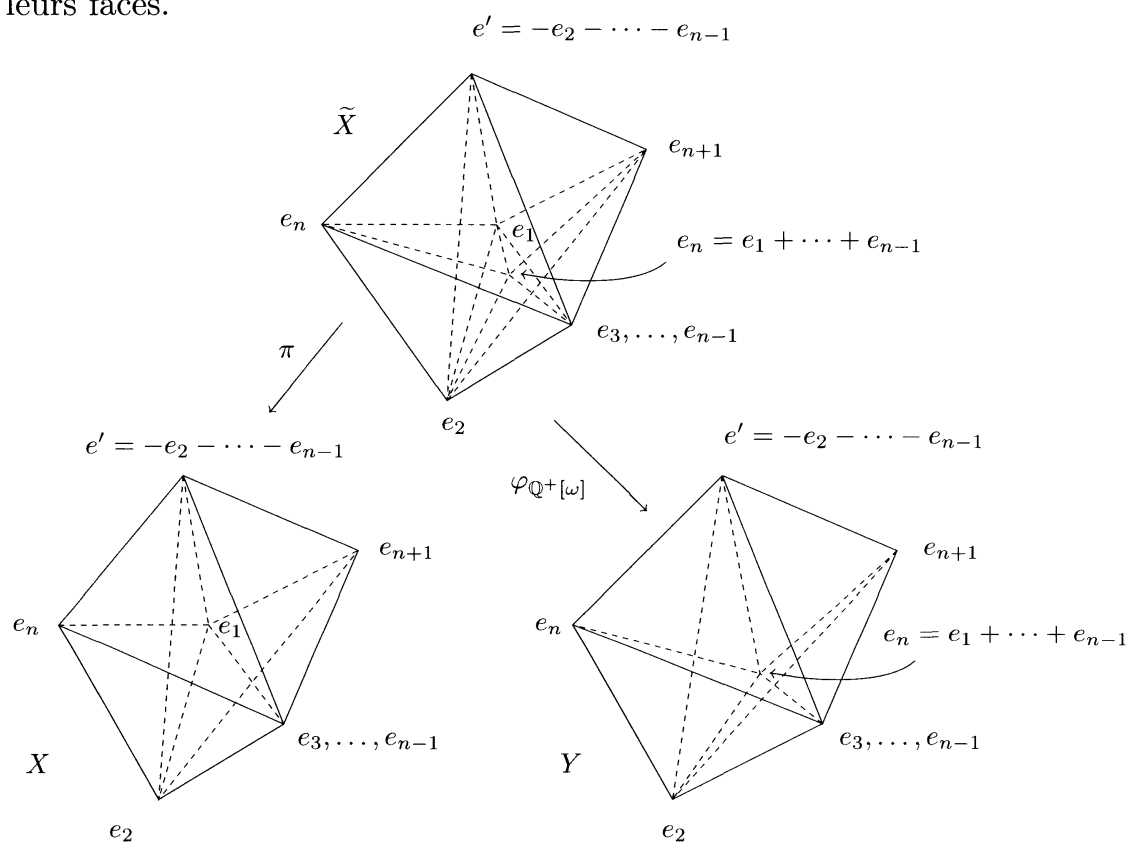

Figure 6. Transformation élémentaire.

Autrement dit, $\varphi_{\mathbb{Q}^{+}[\omega]}: \widetilde{X} \rightarrow Y$ est l'éclatement de $Y$ le long de la sous-variété torique de codimension 2 définie par le cône $\left\langle e, e^{\prime}\right\rangle$. De plus, dans l'éventail $\Delta_{Y}$ définissant $Y$, l'étoile de $e$ est constituée des $2 n-2$ cônes maximaux

$$
\begin{gathered}
\left\langle e_{2}, \ldots, \widehat{e_{i}}, e_{n-1}, e^{\prime}, e, e_{n}\right\rangle, \quad\left\langle e_{2}, \ldots, \widehat{e}_{i}, e_{n-1}, e^{\prime}, e, e_{n+1}\right\rangle, \quad 2 \leq i \leq n-1, \\
\left\langle e_{2}, \ldots, e_{n-1}, e, e_{n}\right\rangle, \quad\left\langle e_{2}, \ldots, e_{n-1}, e, e_{n+1}\right\rangle
\end{gathered}
$$

et leurs faces. En particulier, la forme linéaire $\lambda: N_{\mathbb{Q}} \rightarrow \mathbb{Q}$ définie par $\lambda(e)=\lambda\left(e_{i}\right)=0$ si $2 \leq i \leq n-1$ et $\lambda\left(e_{n}\right)=1\left(\right.$ et donc $\lambda\left(e_{n+1}\right)=-1$ !) induit un morphisme surjectif de l'éventail de l'étoile de $e$ dans $\Delta_{Y}$ sur l'éventail de $\mathbb{P}^{1}$ et donc induit une fibration torique lisse du diviseur torique $E$ défini par

TOME $128-2000-\mathrm{N}^{\circ} 3$ 
le cône $\langle e\rangle$ sur $\mathbb{P}^{1}$, à fibre isomorphe à $\mathbb{P}^{n-2}$. Enfin, comme

$$
e^{\prime}+e_{2}+\sum_{i=3}^{n-1} e_{i}=0
$$

la courbe rationnelle $\left\langle e_{3}, \ldots, e_{n-1}, e, e_{n}\right\rangle$ (incluse dans une fibre de $\lambda$ ) a pour fibré normal dans $Y$ le fibré $\mathcal{O}^{\oplus 2} \oplus \mathcal{O}(1)^{\oplus n-3}$ et le fibré normal $N_{E / Y}$ est donc trivial en restriction aux fibres de $\lambda$. Tout ceci achève de montrer que $X$ est obtenue via une transformation élémentaire de la variété torique projective $Y$.

Remarque. - Dans la situation précédente, on a un diagramme :

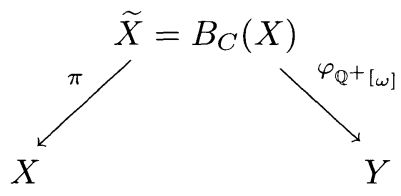

où les deux flèches $\pi$ et $\varphi_{\mathbb{Q}^{+}}[\omega]$ sont des éclatements le long de centres lisses. En fait, on peut itérer la construction précédente : éclatons $Y$ le long de la courbe de cône $\left\langle e_{2}, \ldots, e_{n-1}, e\right\rangle$, la variété projective alors obtenue est elle-même l'éclatement le long d'un centre lisse de codimension 2 dans une autre variété torique lisse, et on peut recommencer. Ainsi $X$ fait partie d'une suite infinie de variétés toriques lisses, s'obtenant successivement à l'aide de transformations élémentaires. Nous ne savons pas déterminer de façon systématique le caractère projectif ou non de ces variétés. Nous verrons dans la partie 4 deux exemples de comportements distincts.

3.2. Le cas où $\boldsymbol{E} \cdot \boldsymbol{\omega}<\mathbf{0}$. - On démontre la proposition suivante :

Proposition 2. - Soit $X$ une variété torique non projective. Supposons que:

(i) il existe une courbe $C$ torique dans $X$ de sorte que $\widetilde{X}:=B_{C}(X)$ est projective;

(ii) il existe une courbe torique $\omega$ de $\widetilde{X}$ Mori extrémale vérifiant $E \cdot \omega<0$ où $E$ est le diviseur exceptionnel de $\pi: \widetilde{X} \rightarrow X$.

Alors,

$$
\left(C, N_{C / X}\right) \simeq\left(\mathbb{P}^{1}, \mathcal{O}_{\mathbb{P}^{1}}(-1)^{\oplus n-1}\right)
$$

et il y a un diagramme

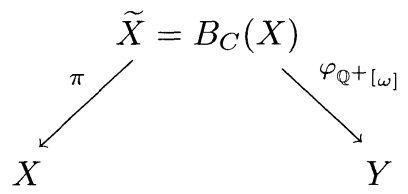

BULLETIN DE LA SOCIÉTÉ MATHÉMATIQUE DE FRANCE 
où $Y$ est une variété torique projective contenant une sous-variété torique $Z$ vérifiant

$$
\left(Z, N_{Z / Y}\right) \simeq\left(\mathbb{P}^{n-2}, \mathcal{O}_{\mathbb{P}^{n-2}}(-1)^{\oplus 2}\right),
$$

$\varphi_{\mathbb{Q}^{+}[\omega]}$ est la contraction extrémale définie par $\omega$ et l'éclatement de $Y$ le long de la sous-variété $Z$ de sorte que $\varphi_{\mathbb{Q}^{+}[\omega]}: \widetilde{X} \rightarrow Y$ et $\pi: \widetilde{X} \rightarrow X$ ont même diviseur exceptionnel $E$ vérifiant

$$
\left(E, N_{E / \widetilde{X}}\right) \simeq\left(\mathbb{P}^{1} \times \mathbb{P}^{n-2}, \mathcal{O}_{\mathbb{P}^{1} \times \mathbb{P}^{n-2}}(-1,-1)\right) .
$$

Démonstration. - Comme $E \cdot \omega<0$, la courbe $\omega$ est incluse dans $E$. Mais comme $X$ n'est pas projective, le lemme 1 implique que $\omega$ n'est pas incluse dans une fibre de $\pi$. Quitte à renuméroter les $e_{i}, 1 \leq i \leq n-1$, on peut supposer que $\omega$ est définie par le mur $\left\langle e_{1}, \ldots, e_{n-2}, e\right\rangle$ et on a une relation

$$
e_{n}+e_{n+1}+\sum_{i=1}^{n-2} a_{i} e_{i}+a e=0,
$$

où les $a_{i}$ et $a$ sont des entiers. Le lemme suivant est l'analogue du lemme 4 .

Lemme 5. - Les entiers $a_{i}$ sont négatifs ou nuls pour tout $i$ entre 1 et $n-2$.

Démonstration. - Sinon, on a par exemple $a_{1}>0$, et d'après [Rei83, cor. 2.10 (i)], les deux courbes $\omega^{\prime}:=\left\langle e_{2}, \ldots, e_{n-2}, e, e_{n}\right\rangle$ et $\omega^{\prime \prime}:=\left\langle e_{2}, \ldots, e_{n-2}, e, e_{n+1}\right\rangle$ sont sur l'arête extrémale $\mathbb{Q}^{+}[\omega]$. Or ces deux courbes sont contractées par $\pi$, ce qui n'est pas possible puisque $X$ est non projective.

Fin de la démonstration de la proposition 2. - Comme

$$
-K_{\widetilde{X}} \cdot \omega=2+\sum_{i=1}^{n-2} a_{i}+a>0
$$

avec $a=E \cdot \omega<0$, il s'ensuit que $a=-1$ et $a_{i}=0$ pour tout $1 \leq i \leq n-2$. La contraction extrémale $\varphi_{\mathbb{Q}^{+}[\omega]}$ est donc divisorielle, de fibres non triviales isomorphes à $\mathbb{P}^{1}$, et comme

$$
e_{n}+e_{n+1}=e=\sum_{i=1}^{n-1} e_{i},
$$

la courbe $C$ a son fibré normal isomorphe à $\mathcal{O}_{\mathbb{P}^{1}}(-1)^{\oplus n-1}$, et donc

$$
\left(E, N_{E / \tilde{X}}\right) \simeq\left(\mathbb{P}^{1} \times \mathbb{P}^{n-2}, \mathcal{O}_{\mathbb{P}^{1} \times \mathbb{P}^{n-2}}(-1,-1)\right) .
$$

Il en résulte - voir la figure 7 - que la contraction $\varphi_{\mathbb{Q}^{+}[\omega]}$ envoie $\tilde{X}$ sur la variété projective lisse $Y$ dont l'éventail est obtenu en remplaçant dans $\widetilde{\Delta}$ l'étoile

TOME $128-2000-\mathrm{N}^{\circ} 3$ 


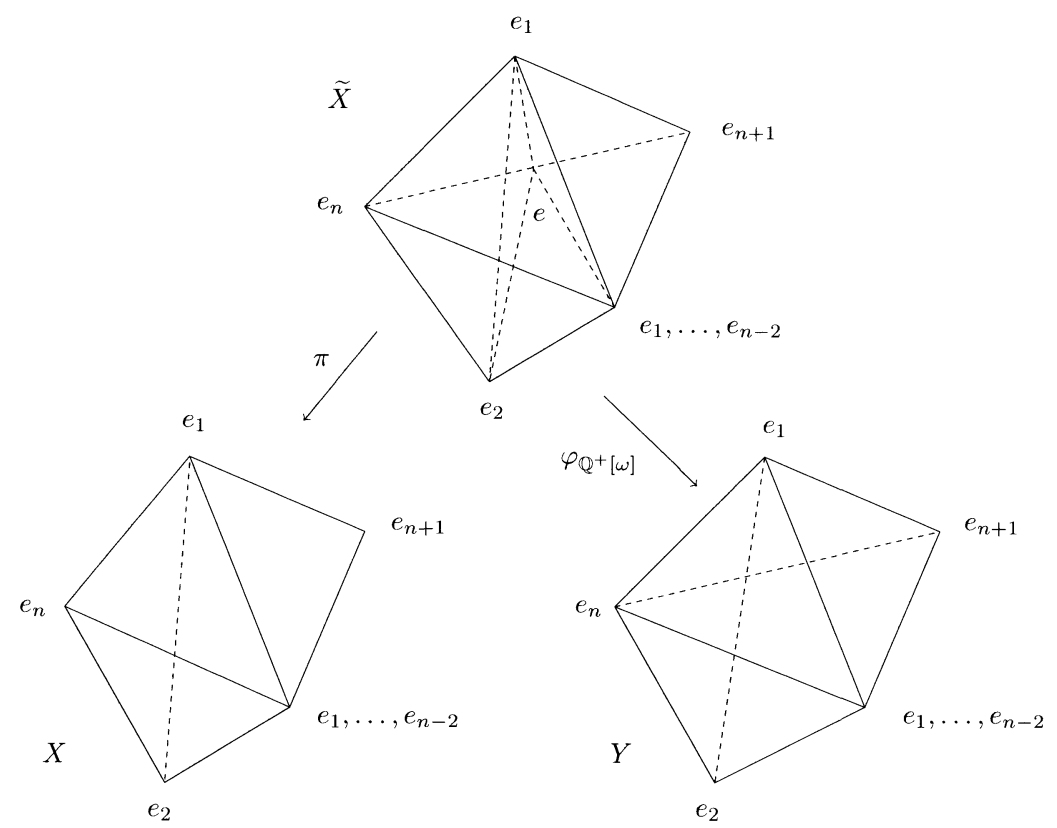

Figure 7. Flip interdit

de $e$ par la réunion des $n-1$ cônes maximaux

$$
\left\langle e_{1}, \ldots, \widehat{e}_{i}, \ldots, e_{n-1}, e_{n}, e_{n+1}\right\rangle, \quad 1 \leq i \leq n-1
$$

et leurs faces.

Autrement dit, $\varphi_{\mathbb{Q}^{+}[\omega]}: \widetilde{X} \rightarrow Y$ est l'éclatement de $Y$ le long de la sous-variété torique de codimension 2 , isomorphe à $\mathbb{P}^{n-2}$ et définie par le cône $\left\langle e_{n}, e_{n+1}\right\rangle$.

\section{Quelques exemples}

Dans cette dernière partie, nous donnons des exemples explicites de variétés toriques non projectives pour toutes les situations précédemment étudiées.

\subsection{Exemples en dimension 3.}

4.1.1. Flip interdit. - L'exemple le plus célèbre est donné par la seule variété torique $X$ non projective de dimension 3 dont le groupe de Picard est de rang 4 : son éventail est donné par la figure 8 projetée sur la face $\left\langle n_{1}, n_{2}, n_{3}\right\rangle$ (voir [Oda88, p. 85]), où $n_{1}, n_{2}$ et $n_{3}$ forment une base du réseau $N$, où $n_{0}=-n_{1}-n_{2}-n_{3}$ et pour $1 \leq i \leq 3, n_{i}^{\prime}=n_{0}+n_{i}$. 


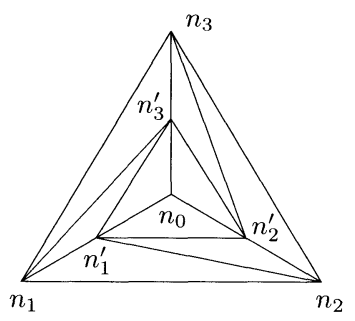

Figure 8

Si $C_{1}$ (respectivement $C_{2}, C_{3}$ ) est la courbe de cône $\left\langle n_{2}^{\prime}, n_{3}^{\prime}\right\rangle$ (respectivement $\left.\left\langle n_{1}^{\prime}, n_{3}^{\prime}\right\rangle,\left\langle n_{1}^{\prime}, n_{2}^{\prime}\right\rangle\right)$, alors $N_{C_{i} / X}=\mathcal{O}_{\mathbb{P}^{1}}(-1)^{\oplus 2}$, la variété $\widetilde{X_{i}}=B_{C_{i}}(X)$ est projective et $X$ est obtenue via un flip interdit d'une variété torique projective $Y_{i}$.

4.1.2. Transformation élémentaire. - D'après la classification [Oda78], [Oda88], des variétés toriques de dimension 3 avec petit groupe de Picard, les variétés toriques non projectives de dimension 3 obtenues via une transformation élémentaire d'une variété torique projective ont un groupe de Picard de rang supérieur ou égal à 5 . L'éventail le plus simple est celui de la variété $X_{a, b}$ suivante $\left(a\right.$ et $b$ dans $\mathbb{Z}$ ) avec $\rho\left(X_{a, b}\right)=5$ (labellée $\left(4^{4} 5^{4}\right)^{\prime}$ dans [Oda88, p. 64] et $\left[8-13^{\prime}\right]$ dans $[$ Oda78, p. 79]).

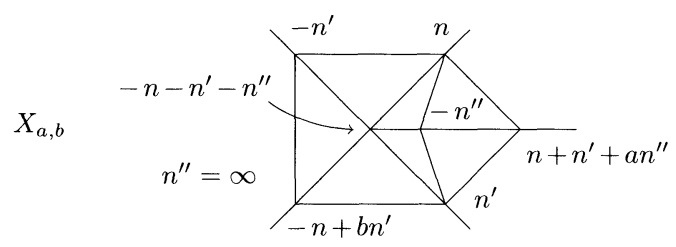

Figure 9

Le résultat suivant est énoncé sans démonstration dans [Oda78, p. 80].

Proposition 3. - La variété $X_{a, b}$ est non projective si et seulement si $a \neq 0$ et $b \neq-1$.

Démonstration. - Si par exemple $a=0$ (le cas $b=-1$ se traite de façon identique), $X_{0, b}$ est projective car obtenue en éclatant un point puis une courbe à partir d'une variété $Z$ projective (le groupe de Picard de $Z$ est de rang 3 ou plus simplement $Z$ admet une fibration sur $\mathbb{P}^{1}$ de fibre $\left.\mathbb{P}\left(\mathcal{O}_{\mathbb{P}^{1}} \oplus \mathcal{O}_{\mathbb{P}^{1}}(b)\right)\right)$.

Supposons ensuite $a \geq 1$ et $b \geq 0$ et montrons que $X_{a, b}$ est non projective (les trois autres cas se traitent de façon identique). Par l'absurde, si $X_{a, b}$ est projective, il existe une fonction $\varphi: N_{\mathbb{Q}} \rightarrow \mathbb{R}$ linéaire sur chaque cône de l'éventail

TOME $128-2000-\mathrm{N}^{\circ} 3$ 
$\Delta$ définissant $X_{a, b}$ et strictement convexe au sens où $\varphi\left(n_{1}+n_{2}\right)>\varphi\left(n_{1}\right)+\varphi\left(n_{2}\right)$ dès que $n_{1}$ et $n_{2}$ n'appartiennent pas à un même cône de $\Delta$. Écrivons alors

$$
\left(-n+b n^{\prime}\right)+(b+1)\left(-n^{\prime}\right)=-n-n^{\prime}=n^{\prime \prime}+\left(-n-n^{\prime}-n^{\prime \prime}\right) .
$$

Comme $n^{\prime \prime}$ et $-n-n^{\prime}-n^{\prime \prime}$ n'appartiennent pas à un même cône alors que $-n+b n^{\prime}$ et $(b+1)\left(-n^{\prime}\right)$ appartiennent à $\left\langle-n+b n^{\prime},-n^{\prime}\right\rangle$, on a :

$$
\varphi\left(-n+b n^{\prime}\right)+(b+1) \varphi\left(-n^{\prime}\right)>\varphi\left(n^{\prime \prime}\right)+\varphi\left(-n-n^{\prime}-n^{\prime \prime}\right) .
$$

De même, $\left(-n-n^{\prime}-n^{\prime \prime}\right)+(b+1) n^{\prime}=-n^{\prime \prime}+\left(-n+b n^{\prime}\right)$ donne

$$
\varphi\left(-n-n^{\prime}-n^{\prime \prime}\right)+(b+1) \varphi\left(n^{\prime}\right)>\varphi\left(-n^{\prime \prime}\right)+\varphi\left(-n+b n^{\prime}\right) .
$$

Par somme :

$$
(b+1)\left(\varphi\left(n^{\prime}\right)+\varphi\left(-n^{\prime}\right)\right)>\varphi\left(n^{\prime \prime}\right)+\varphi\left(-n^{\prime \prime}\right) .
$$

À nouveau,

$$
\left(n+n^{\prime}+a n^{\prime \prime}\right)+a\left(-n^{\prime \prime}\right)=n+n^{\prime} \quad \text { et } \quad n+a n^{\prime \prime}=\left(n+n^{\prime}+a n^{\prime \prime}\right)+\left(-n^{\prime}\right)
$$

donnent

$$
\begin{aligned}
& \varphi\left(n+n^{\prime}+a n^{\prime \prime}\right)+a \varphi\left(-n^{\prime \prime}\right)>\varphi(n)+\varphi\left(n^{\prime}\right), \\
& \varphi(n)+a \varphi\left(n^{\prime \prime}\right)>\varphi\left(n+n^{\prime}+a n^{\prime \prime}\right)+\varphi\left(-n^{\prime}\right),
\end{aligned}
$$

d'où

$$
a\left(\varphi\left(n^{\prime \prime}\right)+\varphi\left(-n^{\prime \prime}\right)\right)>\varphi\left(n^{\prime}\right)+\varphi\left(-n^{\prime}\right)
$$

En regroupant,

$$
a(b+1)\left(\varphi\left(n^{\prime \prime}\right)+\varphi\left(-n^{\prime \prime}\right)\right)>\varphi\left(n^{\prime \prime}\right)+\varphi\left(-n^{\prime \prime}\right),
$$

et comme $0>\varphi\left(n^{\prime \prime}\right)+\varphi\left(-n^{\prime \prime}\right)$, on en déduit $a(b+1)<0$, ce qui est absurde.

La proposition suivante est immédiate :

Proposition 4. - Pour tous a et b entiers, les variétés $X_{a-1, b}, X_{a+1, b}, X_{a, b-1}$ et $X_{a, b+1}$ sont obtenues via une transformation élémentaire de la variété $X_{a, b}$.

Démonstration. - La figure 10 est suffisante.

Des deux propositions précédentes se déduit immédiatement le : 


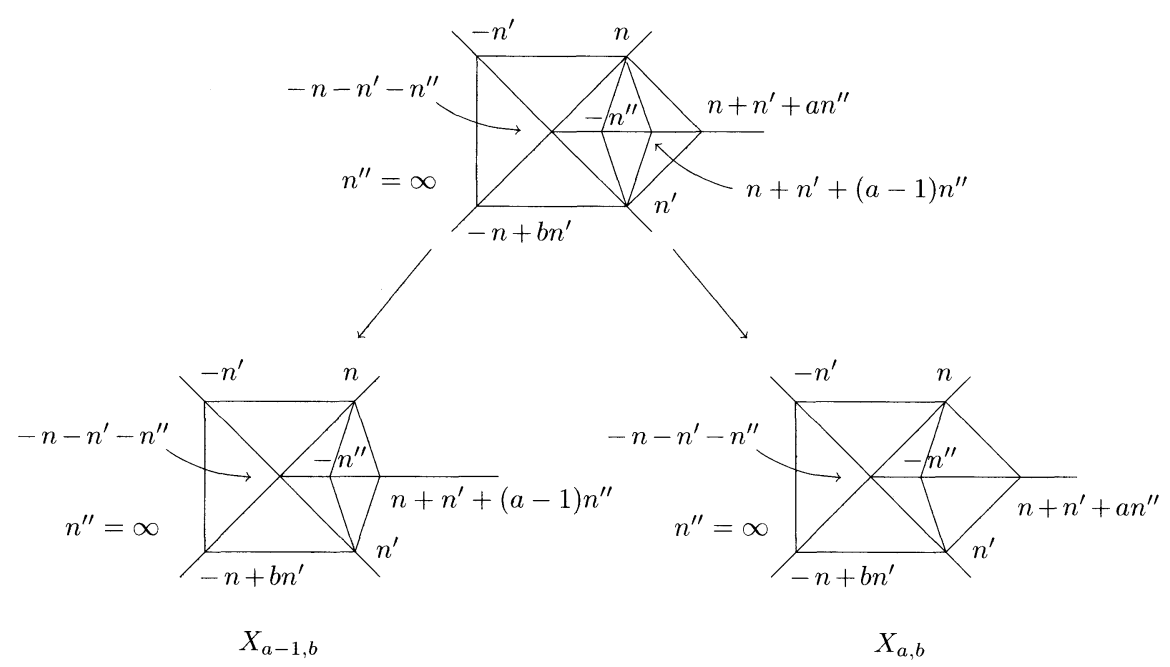

Figure 10

Corollaire 3. - Pour $b \neq-1$, les variétés toriques non projectives $X_{1, b}$ et $X_{-1, b}$ sont obtenues via une transformation élémentaire de la variété torique projective $X_{0, b}$. Pour $a \neq 0$, les variétés toriques non projectives $X_{a, 0}$ et $X_{a,-2}$ sont obtenues via une transformation élémentaire de la variété torique projective $X_{a,-1}$.

4.2. Des exemples en dimensions supérieures : une construction d'Ewald revisitée. - Dans cette dernière partie, nous étudions géométriquement et généralisons une construction due à Ewald [Ewa86] pour construire en toutes dimensions supérieures à 4 des variétés toriques non projectives avec petit groupe de Picard.

4.2.1. Description géométrique, cas général. - Soit $X$ une variété analytique complexe (non nécessairement torique) de dimension $n$ munie d'une action de $\mathbb{C}^{*}$. On construit une variété $\tilde{X}$ de dimension $n+1$ en recollant $\mathbb{C} \times X$ à $\mathbb{P}^{1} \backslash\{0\} \times X$ sur l'ouvert commun $\mathbb{C}^{*} \times X$ grâce à l'action de $\mathbb{C}^{*}:(t, x) \in \mathbb{C}^{*} \times X \subset \mathbb{C} \times X$ est identifié à $(t, t \cdot x) \in \mathbb{C}^{*} \times X \subset \mathbb{P}^{1} \backslash\{0\} \times X$. La variété $\widetilde{X}$ possède par construction une fibration $f: \widetilde{X} \rightarrow \mathbb{P}^{1}$ dont toutes les fibres sont isomorphes à $X$. Dans la suite, on notera $X_{0}:=f^{-1}(0)$ et $X_{\infty}:=f^{-1}(\infty)$. Remarquons qu'à toute sousvariété $Y$ de $X$, stable par $\mathbb{C}^{*}$, correspond une sous-variété $\widetilde{Y}$ de $\widetilde{X}$ de même codimension que $Y$ obtenue par le recollement de $\mathbb{C} \times Y$ à $\mathbb{P}^{1} \backslash\{0\} \times Y$. Comme $\tilde{X}$, $\widetilde{Y}$ possède une fibration sur $\mathbb{P}^{1}$, de fibre isomorphe à $Y$. De plus,

$$
\tilde{Y} \cap X_{0} \simeq \tilde{Y} \cap X_{\infty} \simeq Y \text {. }
$$

TOME $128-2000-\mathrm{N}^{\circ} 3$ 
4.2.2. Description géométrique, cas torique. - Si $X$ est une variété torique, et si $v$ est un élément du réseau $N$, on note classiquement $\lambda_{v}$ le sous-groupe à un paramètre du tore $\operatorname{Hom}\left(M, \mathbb{C}^{*}\right)$ lui correspondant. Ce sous-groupe définit une action de $\mathbb{C}^{*}$ sur $X$ si bien que la construction précédente s'applique : on note $\widetilde{X}_{v}$ la variété correspondante et $f_{v}: \widetilde{X}_{v} \rightarrow \mathbb{P}^{1}$ la fibration correspondante. Comme les actions sur $X$ de $\operatorname{Hom}\left(M, \mathbb{C}^{*}\right)$ et de $\lambda_{v}$ commutent, elles induisent une action de $\operatorname{Hom}\left(M, \mathbb{C}^{*}\right) \times \mathbb{C}^{*}$ sur $\widetilde{X}_{v}$, faisant de cette variété une variété torique de dimension $n+1$ possédant une fibration $f_{v}$ équivariante lorsque $\mathbb{P}^{1}$ est muni de l'action standard de $\mathbb{C}^{*}$. Dans ce cas, $X_{0}$ et $X_{\infty}$ sont deux sousvariétés toriques de $\widetilde{X}_{v}$ et si $Y$ est une sous-variété torique de $\underset{\widetilde{X}}{X}$, la sous-variété $\widetilde{Y}$ de $\widetilde{X}_{v}$ lui correspondant est torique. De plus, il y a dans $\widetilde{X}_{v}$ une sous-variété torique privilégiée, que l'on notera $\widetilde{Y}_{v}$ : en effet $v$ appartient à l'intérieur relatif d'un unique cône $\sigma_{v}$ de l'éventail $\Delta$ et la sous-variété torique $Y_{v}$ de $X$ déterminée par $\sigma_{v}$ est fixée par le sous-groupe $\lambda_{v}$. Par conséquent, la sous-variété torique de $\widetilde{X}_{v}$ lui correspondant est isomorphe à $\mathbb{P}^{1} \times Y_{v}$.

4.2.3. Description "éventail". — Si $\Delta$ est l'éventail dans $N_{\mathbb{Q}}$ de $X$, l'éventail $\widetilde{\Delta}_{v}$ dans $N_{\mathbb{Q}} \oplus \mathbb{Q}$ de $\widetilde{X}_{v}$ est défini de la façon suivante :

- on identifie $\Delta$ avec son image via l'inclusion $N_{\mathbb{Q}} \oplus 0 \hookrightarrow N_{\mathbb{Q}} \oplus \mathbb{Q}$,

- les cônes de $\widetilde{\Delta}_{v}$ sont exactement les

$$
\widetilde{\sigma}:=(\sigma, 0), \quad \tilde{\sigma}_{+}:=(\sigma, 0)+\mathbb{Q}^{+}(v, 1) \quad \text { et } \quad \tilde{\sigma}_{-}:=(\sigma, 0)+\mathbb{Q}^{+}(0,-1)
$$

où $\sigma$ décrit l'ensemble des cônes de $\Delta$.

4.2.4. La construction d'Ewald. - Soit $X$ une variété torique de dimension $n$, $D$ un diviseur irréductible torique de $X$. On note $v_{D}$ le générateur minimal dans $N$ du cône de dimension 1 définissant $D$. D'après ce qui précède, la variété torique $\widetilde{X}_{v_{D}}$ de dimension $n+1$ construite en $4.2 .1-2$ contient un diviseur torique noté $\widetilde{D}$ isomorphe à $\mathbb{P}^{1} \times D$ et Ewald remarque que le fibré normal $\mathcal{N}$ de $\widetilde{D}$ dans $\widetilde{X}_{v_{D}}$ satisfait

$$
\mathcal{N}_{\mid \mathbb{P}^{1} \times\{*\}} \simeq \mathcal{O}_{\mathbb{P}^{1}}(-1)
$$

Par conséquent, il y a une variété torique $X_{v_{D}}$ de dimension $n+1$ contenant une sous-variété torique $Z_{v_{D}}$ de codimension 2 isomorphe à $D$ de sorte que $\widetilde{X}_{v_{D}}$ soit l'éclatement de $X_{v_{D}}$ le long de $Z_{v_{D}}$, le diviseur exceptionnel de l'éclatement étant égal à $\widetilde{D}$. Remarquons que $X_{v_{D}}$ contient deux sous-variétés toriques de codimension 1 isomorphes à $X_{0}$ et $X_{\infty}$, dont l'intersection est isomorphe à $D$.

Le résultat suivant est dû à Ewald :

ThÉorème 2. - Soit $X$ une variété torique, $D$ un diviseur torique de $X$ et $X_{v_{D}}$ la variété torique construite précédemment. Alors $X_{v_{D}}$ est projective si et seulement si $X$ est projective. De plus, $X$ et $X_{v_{D}}$ ont même nombre de Picard. 
D'après ce qui précède, seule l'affirmation « $X$ projective implique $X_{v_{D}}$ projective » est non triviale, Ewald la démontre en considérant l'éventail définissant $X_{v_{D}}$. Ceci peut aussi se démontrer facilement en utilisant notre critère de projectivité $\S 1.4$.

4.2.5. Applications. - Soit $X$ une variété torique non projective de dimension $n$ devenant projective après éclatement le long d'une sous-variété torique $Y$. On note $X^{\prime}:=B_{Y}(X)$. Deux constructions différentes sont possibles :

(i) Si $D$ est un diviseur irréductible torique de $X$ disjoint de $Y$, alors la variété torique $X_{v_{D}}$ de dimension $n+1$ est non projective et devient projective après éclatement le long de la sous-variété torique $\widetilde{Y}$ correspondant à $Y$. Par construction, la codimension de $Y$ dans $X$ est égale à celle de $\widetilde{Y}$ dans $X_{v_{D}}$.

(ii) Si $D$ est un diviseur irréductible torique de $X$ contenant $Y$, la transformée stricte de $D$ dans $X^{\prime}=B_{Y}(X)$ étant notée $D^{\prime}$, alors la variété torique $X_{v_{D}}$ de dimension $n+1$ est non projective et devient projective après éclatement le long d'une sous-variété torique isomorphe à $Y$ (et que l'on note encore $Y$ ) incluse dans $Z_{v_{D}}$. En effet, on a $B_{Y}\left(X_{v_{D}}\right)=X_{v_{D^{\prime}}}^{\prime}$ et cette dernière variété est projective puisque $X^{\prime}$ l'est. Par construction, $X$ et $X_{v_{D}}$ deviennent projectives après éclatement le long de sous-variétés isomorphes, donc de même dimension!

On en déduit le corollaire suivant :

Corollaire 4. - Pour tout entier $n \geq 3$, il existe une variété torique non projective, dont le nombre de Picard est égal à 4, devenant projective après éclatement le long d'une courbe torique.

Démonstration. - Il suffit d'itérer la construction expliquée en (ii) à partir de la variété décrite en 4.1.1.

Les variétés du corollaire 4 sont obtenues via un flip interdit d'une variété torique projective, la même construction partant par exemple des variétés $X_{1, b}$ $(b \neq-1)$ de 4.1 .2 permet de construire, pour tout entier $n \geq 3$, une variété torique non projective, dont le nombre de Picard est égal à 5 , obtenue via une transformation élémentaire d'une variété torique projective.

\section{BIBLIOGRAPHIE}

[Bat82] Batyrev (V.V.). - Toroidal Fano 3-folds, Math. USSR, Izv., t. 19, 1982, p. $13-25$.

[Bon95] Bonavero (L.). - Inégalités de Morse et variétés de Moishezon, Thèse, Université Joseph Fourier, 1995 .

TOME $128-2000-\mathrm{N}^{\circ} 3$ 
[Bon96] Bonavero (L.). - Sur des variétés de Moishezon dont le groupe de Picard est de rang un, Bull. Soc. Math. France, t. 124, n 3, 1996, p. 503-521.

[Ewa86] Ewald (G.). - Spherical complexes and nonprojective toric varieties, Discrete Comput. Geom., t. 1, 1986, p. 115-122.

[Ewa96] Ewald (G.). - Combinatorial convexity and algebraic geometry, Graduate Texts in Math., Springer, t. 168, 1996.

[Ful93] Fulton (W.). - Introduction to toric varieties., Annals of Math. Studies, Princeton University Press, t. 131, 1993.

[Kle66] Kleiman (S.L.). - Toward a numerical theory of ampleness, Ann. Math., t. 2, ser. 84, 1966, p. 293-344.

[Kle88] Kleinschmidt (P.). - A classification of toric varieties with few generators, Aequationes Math., t. 35, $\mathrm{n}^{\circ} 2 / 3,1988$, p. 254-266.

[KSt91] Kleinschmidt (P.), Sturmfels (B.). - Smooth toric varieties with small Picard number are projective, Topology, t. 30, no 2, 1991, p. 289-299.

[Kol91] Kollár(J.). - Flips, flops, minimal models, Surveys Diff. Geom., t. 1, 1991, p. 113-199.

[Mar82] Maruyama (M.). - Elementary transformations in the theory of algebraic vector bundles, Algebraic geometry, Proc. International Conference, La Rabida/Spain 1981, Lect. Notes Math., t. 961, 1982, p. 241-266.

[Moi67] Moishezon (B.). - On $n$ dimensional compact varieties with $n$ independent meromorphic functions., Amer. Math. Soc. Transl., t. 63, 1967, p. 51-177.

[Mor96] Morelli (R.). - The birational geometry of toric varieties, J. Algebr. Geom., t. 5, $\mathbf{n}^{\circ} \mathbf{4}, 1996$, p. 751-782.

[Oda78] ODA (T.). - Lectures on torus embeddings and applications (based on joint work with Katsuya Miyake), Published for the Tata Institute of Fundamental Research, Bombay, Tata Institute of Fundamental Research Lectures on Mathematics an Physics, Mathematics, Springer-Verlag, t. 57, 1978 .

[Oda88] ODA (T.). - Convex bodies and algebraic geometry : an introduction to the theory of toric varieties, Ergebnisse der Mathematik und ihrer Grenzgebiete, Springer, t. 3, 015, 1988.

[Rei83] REID (M.). - Decomposition of toric morphisms Arithmetic and geometry, dedicated to I.R. Shafarevich, vol. II, Geometry, Prog. Math., t. 36, 1983, p. 395-418.

[WWa82] Watanabe (K.), Watanabe (M.). - The classification of Fano 3-folds with torus embeddings, Tokyo J. Math., t. 5, 1982, p. 37-48. 